\title{
Solution of the problem of impact elastoplastic deformation of a thin layer of mechanoluminophor using the methods of the dislocation microdynamic theory of plasticity
}

\author{
Konstantin Tatmyshevskiy, ${ }^{1, *}$ \\ ${ }^{1}$ Institute of Information Technology and Radio Electronics, Vladimir State University; Gorky Street \\ 87, 600000 Vladimir, Russia
}

\begin{abstract}
The paper presents the results of numerical simulation of the output optical signals of mechanoluminescent shock sensors. Such sensors operate on the principle of direct conversion of mechanical impact energy into optical radiation energy. The sensing element of such a sensor is a thin layer of phosphor enclosed between two transparent flexible polymer films. The mathematical model of the sensor is based on the process of excitation of the glow centers (activator atoms) in a strong electric field of a moving dislocation. The stress-strain state of the film sensing element under quasi-static uniaxial loading under the action of a single pressure pulse is considered. The analysis of constitutive equations for elasto-plastic deformations and the basic equations of the dynamic theory of dislocations. To calculate the deformation of the sensing element, a microscopic model of an isotropic elastic-plastic medium with hardening is used, according to which the plastic deformation is considered as a result of the movement and multiplication of dislocations, and the hardening is as a result of their partial locking due to the increased density.
\end{abstract}

\section{Introduction}

The effectiveness of various technical systems is largely determined by the operation of sensors that supply primary information about the state of the system itself and the external factors affecting it. Expanding the range of product application conditions imposes special requirements on the sensors for noise immunity to electromagnetic interference, speed, reliability, information content, selectivity, miniaturization and the possibility of integration into the product design [1-4].

Mechanoluminescent impulse pressure sensors meet many of the requirements. Such sensors operate on the principle of direct conversion of the mechanical energy of elasticplastic deformation into the energy of optical radiation. Here, the use of output light signals solves the problems of pairing sensors with fiber-optic communication lines and significantly increasing the noise immunity to electromagnetic interference, the absence of

* Corresponding author: tatmysh@mail.ru 
moving parts, the hardness and planarity of the sensor provide reliability, and the use of its spatial modulation, various spectra and polarization state in addition to the amplitude-time parameters of the optical signal increases informational content.

Mechanoluminescent pressure sensors use in their work the phenomenon of luminescence of class $\mathrm{A}_{2} \mathrm{~B}_{6}$ semiconductors that occurs during plastic deformation of semiconductor crystals. The basis for the design of the sensor is a mechanoluminescent transducer in the form of a thin film consisting of fine-grained particles of luminescent powder placed between two adhesive flexible transparent substrates. The optimum thickness of the sensor does not exceed $20 \ldots 100 \mu \mathrm{m}$, which is determined by the particle size distribution of the powder and the characteristics of the propagation of light in finely dispersed layers. The small thickness of mechanoluminescent sensors (MLS) allows the production of miniature flexible films of sensitive surfaces that can be embedded in structural components of products subjected to external mechanical stress.

Successful application of MLS is impossible without the development of physical and mathematical models, simulation mathematical modeling and verification of the adequacy of models through experimental studies of MLS prototypes.

\section{Physical fundamentals of mechanoluminescence}

The physical model of MLS is based on the phenomenon of electron tunneling of impurity glow centres (GC) in the electric field of moving dislocations arising from plastic deformation of a crystal. A known electric model of dislocation, according to which a dislocation, as a linear defect in the crystal lattice, can be represented as a space charge cylinder with a central core filled with electrons [5]. During plastic deformation, dislocations become strained and begin to move within the bulk of the crystal. GC (impurity atoms) are approximately evenly distributed in the bulk of the crystal.

As the core of the dislocation approaches the GC, it moves to a higher energy level. As soon as the rapprochement and overlapping of the main electronic levels with the free levels of the GC or with the conduction band occurs, the processes of electron tunneling from occupied levels to free and into the conduction band become significant. Since the electron tunneling rate is high compared to the dislocation motion velocity, it can be assumed that at the moment of tunneling the GC is removed from the dislocation core by a fixed distance, and tunneling occurs in a constant electric field of the dislocation space charge cylinder [6].

The return of the excited GC to its initial state is accompanied by the emission of a quantum of light. The mathematical model of MLS is an integral equation describing the energy luminosity of a unit area of a sensor $R(t)$ as a function of time

$$
R(t)=\eta \frac{1}{\tau_{L}} \exp \left(-\frac{t}{\tau_{L}}\right) \int_{0}^{t_{\sigma}} N_{G C} 2 r_{i n}(t) \widetilde{N}_{m D}(t) \widetilde{V}_{D}(t) \mathrm{d} t
$$

where $\eta$ - energy of a quantum of light; $\tau_{L}$ - constant time corresponding to the exponential nature of the decay ML; $t$ - current time; $t_{\sigma}$ - the duration of the shock pulse; $N_{G C}$ - total amount of GC in the phosphor; $r_{i n}$ - radius of interaction of a moving dislocation with the GC; $\widetilde{N}_{m D}$ - average density of mobile dislocations; $\widetilde{V}_{D}$ - average velocity of dislocations.

The total number of GCs in the phosphor is determined by the total volume of individual grains of the luminescent material and the volume concentration of GC. The radius of interaction of GC with a moving dislocation $r_{i n}(t)$ strongly depends on the linear dislocation charge density [6] and the current value of the dislocation velocity $\widetilde{V}_{D}(t)$.

Expression (1) describes two contradictory processes, firstly, the product under the integral describes the dynamic process of GC excitation during the action of the pressure 
pulse $t_{\sigma}$, and secondly, the expression in front of the integral describes the process of exponential decay of the glow. This determines the essential dynamic properties of the phenomenon of mechanoluminescence.

However, to calculate the output optical signal, it is necessary to determine the dynamics of plastic deformation of the MLS. Since kinetic dislocation parameters are used in the mathematical model of the light response, it is also desirable to determine the plastic deformation based on dislocation representations.

\section{Equation of quasistatic deformation}

A prerequisite for quasistatic deformation is the uniformity of plastic deformations over the thickness of the sensor. This condition is satisfied either at low strain rates, or at small ratios of the thickness of the sensor to its diameter. The optimal thickness of the sensor (tens of micrometers) is much less than both its diameter (units of millimeters) and the wavelengths of potential pressure pulses $\sigma(t)$. This allows us to neglect the wave nature of the sensor loading, not to take into account the process of pulse decay into elastic and plastic components, and to consider the condition of uniformity of stresses and strains across the sensor thickness to be fulfilled.

We set the inlet pressure in the form of a plane pressure pulse $\sigma(t)$ with the amplitude incident on the sensitive element perpendicular to the plane of its surface. Consider the stress-strain state of MLS. We make the following assumptions: 1) do not take into account the mechanical properties of thin adhesive substrates; 2) consider the sensor as a thin layer of material with isotropic properties, taking into account that the orientation of the crystallographic axes of individual grains of the phosphor is arbitrary. In this case, it is necessary to use not the anisotropic, but the so-called Voigt averaged crystal constants. Such averaging allows us to use the idealization of the isotropy of the crystal [7].

Let us consider the simplest case when the sensitive element has rotation symmetry about the axis of loading. Let us designate this axis 1, and perpendicular to it - 2 and 3 . Axes 1-3 are the main axes of the strain and stress tensors. By virtue of the additivity condition, the general strain tensor is determined in the form of components of the tensors of elastic and plastic strains. The basis of elastic and plastic deformation of bodies with a crystalline structure are various physical processes. The elastic part of the tensor is the result of elastic deformation of atomic lattices, and the plastic part is the result of the movement of dislocations. Therefore, the defining relations connecting the tensors of elastic and plastic strains with the stress tensor are written in two different ways [8]. Elastic deformation obeys Hooke's law. For plastic flow, the postulate of incompressibility is valid $[8,9]$.

We make an additional assumption of uniaxiality of the stress state of the sensing element. The stress $\sigma_{1}$ is applied only along axis 1 . Then the stress-strain state of the sensor can be described as follows

$$
\sigma_{1}=E\left(\varepsilon_{1}-\frac{4}{3} \varepsilon_{p}\right)=\sigma(t)-E \varepsilon_{p 1}, \tau=\frac{\sigma_{1}}{2},
$$

where $E$ - Young's modulus; $\varepsilon_{1}$ - general and plastic $\varepsilon_{\mathrm{p} 1}$ deformation along axis 1 ; $\tau$ - the main value of the shear stress. The physical meaning of equation (2) is that stresses increase due to an increase in the total strain and relax due to plastic deformation. Equation (2) gives a phenomenological explanation of stress relaxation for almost any solid body [7]. The properties of a particular material should be taken into account when choosing a physical model of plastic deformation that determines the value $\varepsilon_{p}$.

The ratio determining $\varepsilon_{p}$. can be set based on dislocation representations. Taylor [9] and Gilman [8] developed a model of elastic-plastic medium in which plastic deformation is 
considered as a result of motion and multiplication of dislocations, and hardening - as a result of their partial locking due to increased density. The convenience of using the dislocation model of plastic deformation of the MLS is that the model is based on the direct determination of the two components necessary to solve the equation (1), describing the kinetics of mechanoluminescent radiation.

Despite the existence in single crystals of certain planes and sliding directions (sliding systems), in polycrystalline structures, which is the structure of MLS, sliding systems in different grains of the phosphor are oriented differently and there are no separate sliding systems. Therefore, it can be assumed [7] that the plane of sliding coincides with the plane of action of the maximum shear stress, which in this case coincides with the plane of maximum plastic deformations. The growth rate of maximum plastic deformations is determined by the Orowan expression [9]

$$
\dot{\varepsilon}_{p}=|\vec{b}| \widetilde{N}_{m D}(t) \widetilde{V}_{D}(t)
$$

where $|\vec{b}|$ - Burgers vector module. It is shown $[8,9]$ that in the process of plastic deformation only a certain part of the total number of dislocations is mobile. In polycrystalline isotropic materials, the total dislocation density can be characterized by an average scalar value. Although the internal structure of the crystal during plastic deformation contains a complex set of forms and sizes of dislocation loops, the behavior of the arbitrary loop is not much different from the behavior of the average loop. Therefore, the behavior of the averaged loop mainly characterizes the behavior of the entire distribution. The relationship between $\widetilde{N}_{t D}$ and $\widetilde{N}_{m D}$ can be described by the expression proposed by Gilman [8]

$$
\widetilde{N}_{m D}=\widetilde{N}_{t D} \exp \left(-\frac{\widetilde{N}_{t D}}{\widetilde{N}_{c r}}\right)
$$

where $\widetilde{N}_{c r}$ - critical value of the total dislocation density corresponding to the end of the flow site in the diagram $\sigma(\varepsilon)$. Formula (4) shows that part of the mobile dislocations is a monotonically decreasing function of the total dislocation density. Moreover, while the total density of dislocations is less than the critical value, with the growth of plastic deformation there is a multiplication of mobile dislocations, and, on the contrary, if the total density of dislocations is less than the critical value, then with the growth of plastic deformation there is a decrease in the density of mobile dislocations, that is, their locking.

Along with the movement of dislocations during plastic deformation their reproduction occurs $[8,9]$. The multiplication of dislocations is due to their movement, and therefore their concentration at the moment depends on their past average speed. Thus, the average dislocation rate plays a primary role in determining the behavior of dislocations in total. Braking forces that cause resistance to dislocation movement are very significant for most crystals. The effective masses of dislocations have the order of one atomic mass per atomic plane. Consequently, dislocations are accelerated to a stationary motion state for times very small compared to loading times. Therefore, the reaction of the material almost always determines the average velocity of the stationary motion. Dislocation multiplication occurs through a process known as multiple sliding. In the process of sliding there are new sources of dislocations. For most solids with a crystalline structure are experimentally confirmed the dependence [8]

$$
\widetilde{N}_{t D}=\widetilde{N}_{t D}^{0}+M \varepsilon_{p}
$$

where $\widetilde{N}_{t D}^{0}$ - average initial dislocation density; $M$ - multiplication factor of the dislocations.

The value in the materials obtained by conventional methods reaches values of $10^{8} \ldots 10^{11} \mathrm{~m}^{-2}$ [7]. The coefficient $M$ for crystals of different structure has the value $\mathrm{M} \sim(0,5 \ldots 1,0) 10^{13} \mathrm{~m}^{-2}[8,9]$. 
The average velocity of dislocations in the expression (3) is very sensitive to shear stresses in the sliding plane and can be described by approximation [8]

$$
\widetilde{V}_{D}=k_{s} c_{t r} \exp \left(\frac{D_{f}}{\sigma_{1}}\right) \text {, }
$$

where $k_{s}$ - the correction factor, which takes into account the presence of a threshold yield stress value below which the motion of dislocations does not occur; $c_{t r}$ - speed of propagation of shear waves in the crystal; $D_{f}=2 \sigma_{s}$ - effective stress internal friction; $\sigma_{s}$ static yield strength.

\section{Results of calculation}

The solution of equations (1) - (6) was found by numerical method in MATLab. Used the solver ode113, which is based on the formula of Adams-Bashforth of Moulton. The quadrature - quad method was used for integration. The results of the solution were substituted into equation (1) to determine the parameters of the output optical signal $R(t)$ of the energy luminosity MLS of a unit area. An example of the simulation results is shown in Fig. 1. Good convergence with experimental data was obtained by modeling $R(t)$ [10].

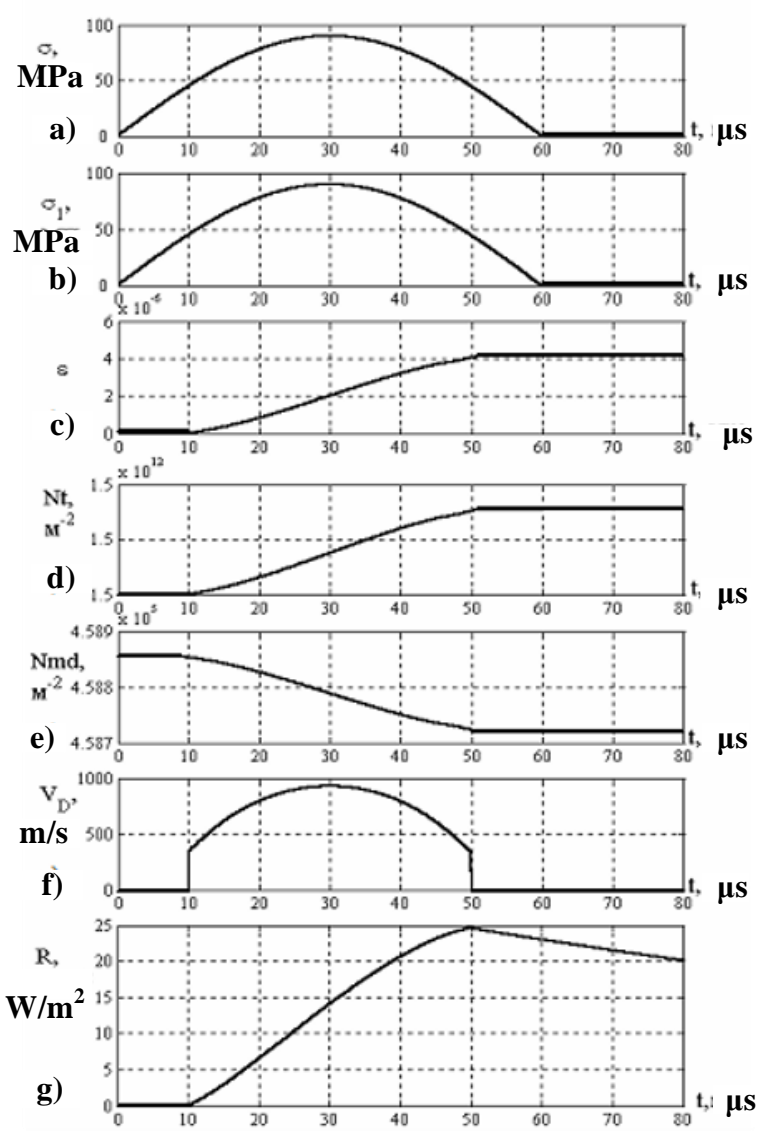

Fig. 1. An example of calculating the dislocation parameters of plastic deformation as a function of time: a - pressure pulse; $b$ - current stress; $\mathrm{c}$ - total deformation; $\mathrm{d}$ - average total density of dislocations; e - average density of moving dislocations; $\mathrm{f}$ - average speed of dislocations; $\mathrm{g}$ - output pulse of energy luminosity (constant attenuation time $\tau=150 \mu \mathrm{s}$; radiation wavelength $\lambda=580 \mathrm{~nm}$ ). 


\section{Summary}

The problem of calculating the kinetics of plastic deformation of a thin crystalline layer of a mechanoluminescent sensor is solved for the case of quasi-static uniaxial loading on the basis of a microscopic model of an isotropic elastoplastic medium with hardening, according to which the plastic deformation is considered as a result of movement and multiplication of dislocations.

The developed model allowed, firstly, to explain the differences in the results of the experiments of various researchers of different dislocation structure of MLS having the same chemical composition, and, secondly, to predict the possibility of multiple increase in the brightness of the glow by reducing the initial density of dislocations

\section{References}

1. A. Feng, S. Michels, A. Lamberti, P.F. Smet, 18th International Conference on Experimental Mechanics (ICEM18), 2, 492 (2018)

2. B.P. Chandra, Mechanoluminescence, in Luminescence of Solids, (New York: Plenum Press, 1998)

3. L.Sodomka, Mechanoluminiscence a jeji pouziti (Praha: Academia,1985)

4. I. Sage, G. Bourhill, J. Mater. Chem. 11, 231 (2001)

5. Z.A.Veliev, V.B. Shikin, Sov. Phys. Sold. St. 27, 2683 (1985)

6. Yu. A. Ossipian et al. Electronic properties of dislocations in semiconductors, (Editorial URSS, 2000)

7. J. P. Hirth, J. Lothe, Theory of dislocations, (2nd ed. John Wiley \& Sons, Inc., 1982)

8. J. J. Gilman, Appl. Mech. Rev. 21, 767 (1968)

9. J. W.Taylor, J. Appl. Phys. 36, 3146 (1965)

10. N.Yu. Makarova, K.V. Tatmyshevsky, Instrum. and Exp. Techn. 49, 135 (2006) 